\title{
Cerebral Venous Thrombosis - Clinical Profile and Role of MRI in Diagnosis and Prognosis
}

\author{
Sandhya $\mathbf{S}^{1}$, Jaiganesh $\mathbf{S}^{2}$, Rajasekhar K $\mathbf{V}^{3}$ \\ ${ }^{1}$ Associate Professor, Department of General Medicine, ${ }^{2}$ Associate Professor, Department of Radiology, ${ }^{3}$ Professor and HOD, \\ Department of Radiology, Meenakshi Medical College Hospital and Research Institute, Kanchipuram, Tamilnadu, India
}

Corresponding author: Dr. Jaiganesh S., No: 5, P. T. Eswaran Street, Arcot - 632 503. Vellore District, Tamilnadu, India

DOI: http://dx.doi.org/10.21276/ijcmsr.2019.4.1.13

How to cite this article: Sandhya S, Jaiganesh S, Rajasekhar K V. Cerebral venous thrombosis - clinical profile and role of MRI in diagnosis and prognosis. International Journal of Contemporary Medicine Surgery and Radiology. 2019;4(1):A53-A57.

\section{A B S T R A C T}

Introduction: Cerebral Venous Thrombosis (CVT) is relatively a rare cause of stroke which is potentially treatable without any permanent neurological deficit if diagnosed early in time. The objective of this study was to describe the etiologies, clinical features and the importance of MRI in diagnosis and prognosis of Cerebral Venous Thrombosis.

Material and Methods: This was a prospective study done in 50 consecutive patients diagnosed with cerebral venous thrombosis on MRI in Meenakshi Medical College Hospital and Research Institute, Kanchipuram between April 2016 and March 2018. Detailed history, proper clinical examination and necessary laboratory investigations were done in all the cases. Results: Among 50 patients, most of the cases were seen in $21-50$ years of age comprising $92 \%$. The most common presenting symptom was headache(82\%) followed by Vomiting(52\%), altered sensorium(44\%) and seizures(42\%). Superior sagittal sinus is the commonest one involved in our study. MRI is helpful to assess the prognosis. Venous infarct is seen in $48 \%$ of the cases and diffusion restriction is seen only in $8 \%$ of cases.

Conclusion: Clinical presentation of Cerebral venous thrombosis(CVT) is vague and high degree of clinical suspicion should be there in young patients presenting with headache, seizures or papilledema. MRI plays a very vital role in the diagnosis and prognosis. Heparin, anticoagulants, antiepileptics and symptomatic treatment play the key role in management. Cerebral venous Thrombosis has very good prognosis provided early diagnosis is made and proper treatment started in time.

Key words: CVT; Venous Infarct; Hemorrhagic Infarct; MR Venogram;

\section{INTRODUCTION}

Cerebral Venous Thrombosis(CVT) was once considered as a rare cause of Stroke, but with recent advances in Imaging Technology and awareness of the disease, diagnosis has been made easy. ${ }^{1}$ Contrary to Arterial Stroke, Cerebral venous Sinus Thrombosis is commonly seen in young adults and accounts for almost $0.5 \%$ of all strokes. ${ }^{2}$ Patients with CVT most commonly present with headache, seizures, vomiting, visual disturbances, loss of consciousness and sometimes with neurological deficits. ${ }^{3}$ MRI and MR Venography are very efficient in detecting Cerebral Venous Thrombosis and the associated parenchymal abnormalities especially the involvement of deep cerebral veins. ${ }^{4}$

The aim of this article was to review the knowledge about clinical features, predisposing factors for Cerebral Venous Thrombosis and the role of MRI in diagnosis and prognosis.

\section{MATERIAL AND METHODS}

This was a prospective descriptive study done in 50 consecutive patients admitted in the Meenakshi Medical College Hospital and Research Institute, Kanchipuram who were diagnosed with Cerebral venous Thrombosis on MRI. Detailed proforma was made which included the complete history, predisposing factors, clinical examination and MRI findings in each patient.

MRI was done in Siemens Essenza $1.5 \mathrm{~T}$ machine with following sequences SE T1 sequence in Axial and Sagittal planes, FSE T2 sequence in Axial and Coronal planes, FLAIR and GRE sequences in Axial plane, TOF MRV sequence in all patients and Contrast Enhanced MR Venography additionally in certain cases.

\section{Inclusion Criteria}

All patients with Cerebral venous sinus/Cortical vein thrombosis confirmed by MRI.

\section{Exclusion Criteria}

Patients with normal Dural Sinuses/Cortical veins on MRI. Patients with MRI Incompatible Devices such as Cochlear Implants, Cardiac Pacemaker etc.

Claustrophobic Patients.

Proper Consent to include in the study was obtained from all the patients. The study was conducted after considering and following all the factors of Declaration of Helsinki.

\section{RESULTS}

Wide range of age groups were affected by cerebral venous thrombosis in our study from $2^{\text {nd }}$ decade to $7^{\text {th }}$ decade in 


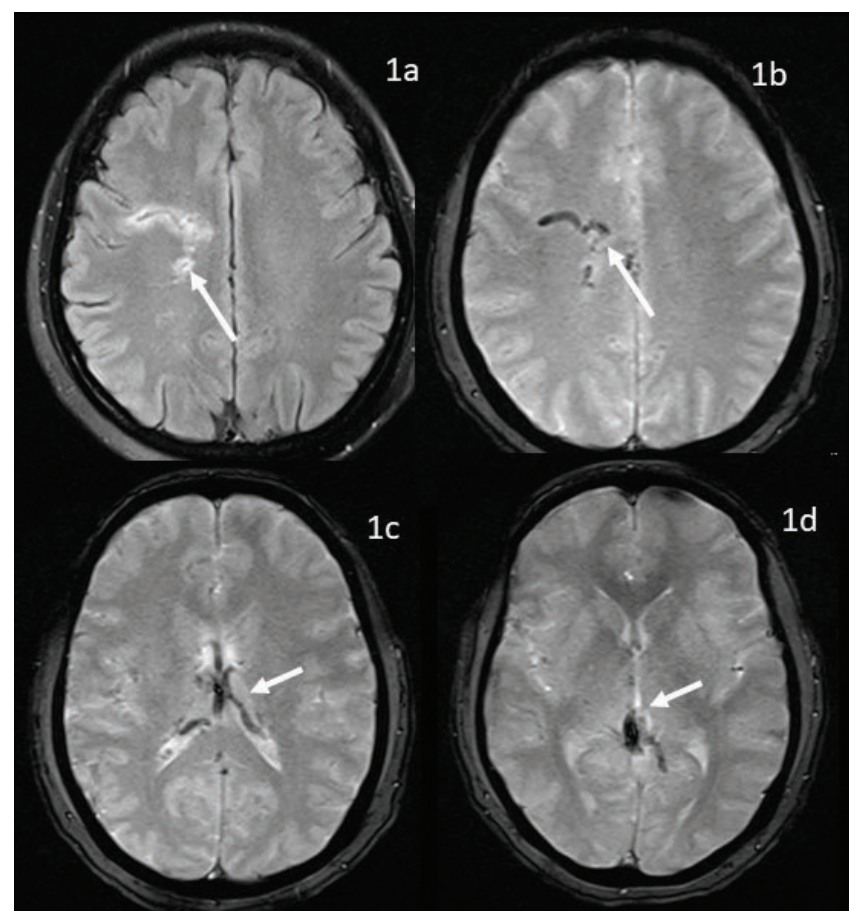

Figure-1: Axial FLAIR (1a) showing infarct (arrow) in right frontal lobe and GRE Axial images (1b, 1c, 1d) showing thrombosed cortical vein and areas of hemorrhages (arrow)

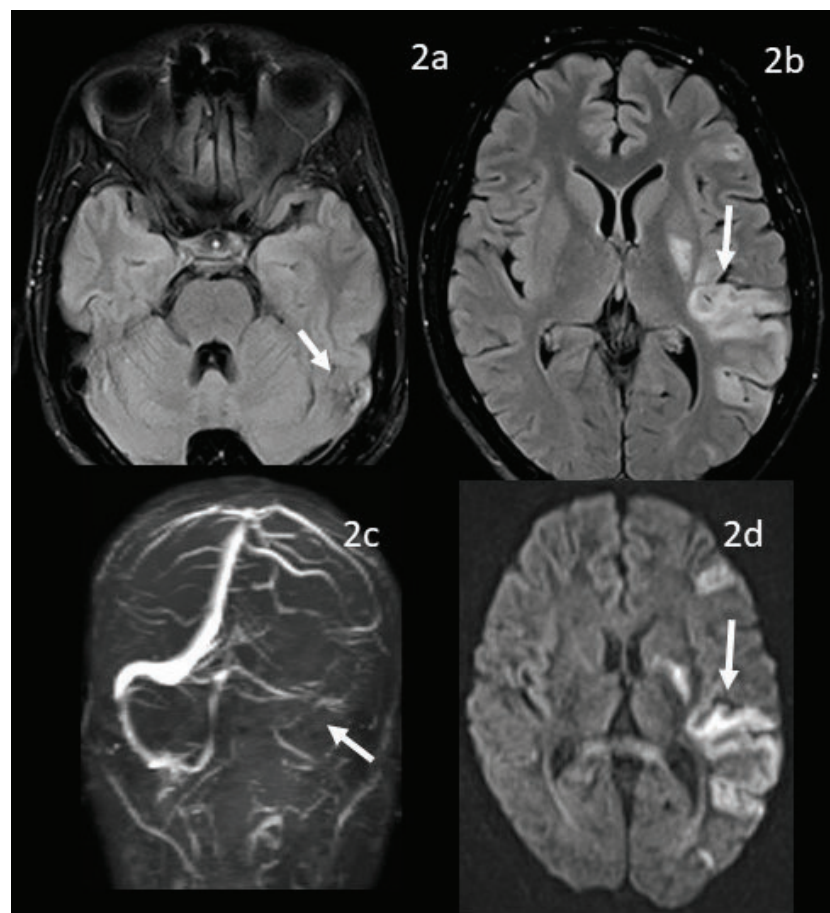

Figure-2: Axial FLAIR showing left sigmoid sinus thrombus (arrow in 2a) and infarct (arrow in 2b) in left temporal lobe and lentiform nucleus. TOF MRV image (2c) shows absent left transverse and sigmoid sinuses. DWI (2d) shows strong restriction

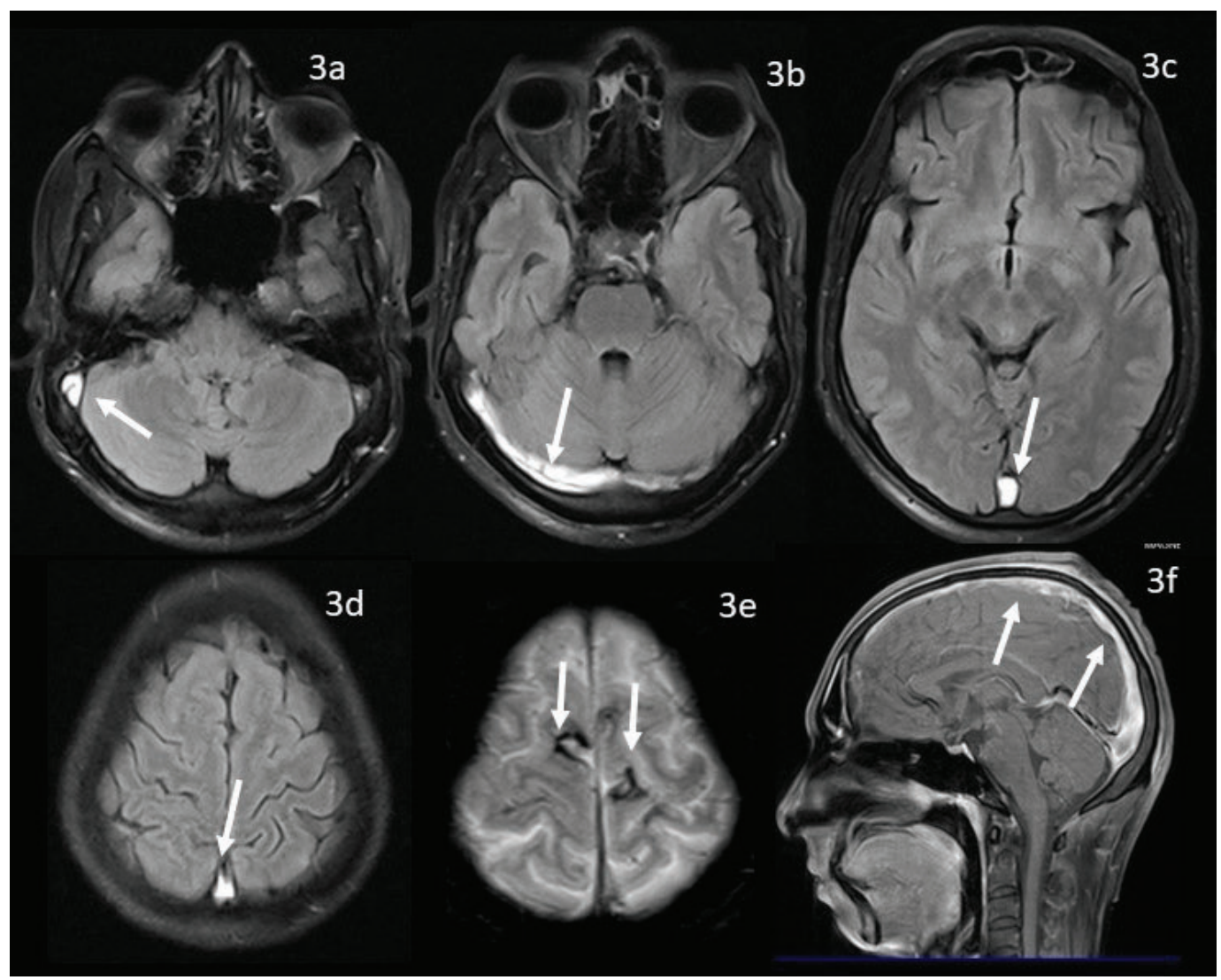

Figure-3: Axial FLAIR images (3a-d) shows thrombus (arrow) in right sigmoid, right transverse adn superior sagittal sinuses. Axial GRE (3e) image shows haemorrhage (arrow) in high frontal lobes and T1 sagittal image shows superior saggittal sinus thrombus (arrow) 


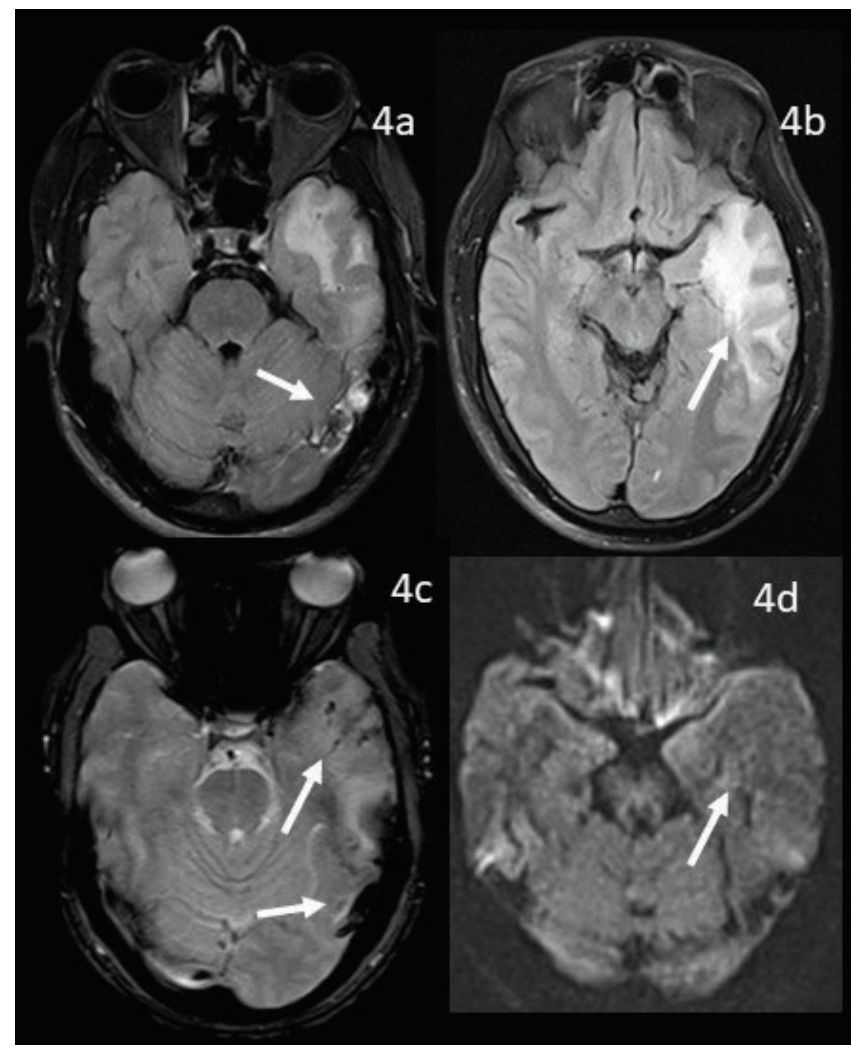

Figure-4: Axial FLAIR showing left sigmoid sinus thrombus (arrow in $4 \mathrm{a}$ ) and infarct (arrow in $4 \mathrm{~b}$ ) in left remporal lobe. GRE axial image (4c) shows hemorrhage. DWI (4d) shows no restriction

\begin{tabular}{|l|c|c|}
\hline Age Group & Number of Cases & Percentage \\
\hline $0-10$ & 0 & 0 \\
\hline $11-20$ & 1 & $2 \%$ \\
\hline $21-30$ & 15 & $30 \%$ \\
\hline $31-40$ & 18 & $36 \%$ \\
\hline $41-50$ & 13 & $26 \%$ \\
\hline $51-60$ & 2 & $4 \%$ \\
\hline $61-70 \quad 1$ & $2 \%$ \\
\hline \multicolumn{2}{|c|}{ Table - 1: Age wise distribution of cases } \\
\hline
\end{tabular}

\begin{tabular}{|l|c|c|}
\hline Sex & Number of Cases & Percentage \\
\hline Male & 21 & $42 \%$ \\
\hline Female & 29 & $58 \%$ \\
\hline \multicolumn{2}{|c|}{ Table-2: Sex wise distribution of Cases } \\
\hline
\end{tabular}

\begin{tabular}{|l|c|c|}
\hline Signs \& Symptoms & Number of Cases & Percentage \\
\hline Headache & 41 & $82 \%$ \\
\hline Vomiting & 26 & $52 \%$ \\
\hline Seizures & 21 & $42 \%$ \\
\hline Altered Sensorium & 22 & $44 \%$ \\
\hline Papilledema & 16 & $32 \%$ \\
\hline Visual Disturbances & 5 & $10 \%$ \\
\hline Neurological Deficits & 13 & $26 \%$ \\
\hline \multicolumn{2}{|c|}{ Table-3: Clinical Signs \& Symptoms } \\
\hline
\end{tabular}

which majority of the patients were seen in 21-40 years of age [Table-1].In our study, there is mild female predilection, about

\begin{tabular}{|l|c|c|}
\hline Risk Factors & $\begin{array}{c}\text { Number of } \\
\text { Cases }\end{array}$ & Percentage \\
\hline Pregnancy/Puerperium & 10 & $20 \%$ \\
\hline Oral Contraceptive Pills & 7 & $14 \%$ \\
\hline Alcohol & 7 & $14 \%$ \\
\hline Dehydration & 6 & $12 \%$ \\
\hline Anemia & 5 & $10 \%$ \\
\hline Infections & 3 & $6 \%$ \\
\hline Connective tissue disorder & 1 & $2 \%$ \\
\hline Thrombophilias & 1 & $2 \%$ \\
\hline Polycythemia & 1 & $2 \%$ \\
\hline Idiopathic Table-4: Risk Factors/Probable Cause \\
\hline \multicolumn{2}{|c|}{} \\
\hline
\end{tabular}

\begin{tabular}{|l|c|c|}
\hline Site & Number of Cases & Percentage \\
\hline Superior Sagittal Sinus & 28 & $56 \%$ \\
\hline Transverse Sinus & 24 & $48 \%$ \\
\hline Sigmoid Sinus & 18 & $36 \%$ \\
\hline Straight Sinus & 15 & $30 \%$ \\
\hline Internal Cerebral Veins & 5 & $10 \%$ \\
\hline Cortical Veins Table-5: Site Involvement \\
\hline \multicolumn{2}{|c|}{. } \\
\hline
\end{tabular}

\begin{tabular}{|l|c|c|}
\hline Parenchymal Changes & $\begin{array}{c}\text { Number of } \\
\text { Cases }\end{array}$ & Percentage \\
\hline Infarcts without Hemorrhage & 8 & $16 \%$ \\
\hline Infarcts with Hemorrhage & 16 & $32 \%$ \\
\hline Diffusion Restriction & 4 & $8 \%$ \\
\hline Nil & 26 & $52 \%$ \\
\hline \multicolumn{2}{|c|}{ Table-6: Parenchymal Changes } \\
\hline
\end{tabular}

\begin{tabular}{|l|c|c|}
\hline Outcome & Number of Cases & Percentage \\
\hline Complete Clinical Recovery & 13 & $65 \%$ \\
\hline Partial Clinical Recovery & 7 & $35 \%$ \\
\hline \multicolumn{2}{|c|}{ Table-7: Clinical Outcome(20 Cases) } \\
\hline
\end{tabular}

$42 \%$ patients were males and $58 \%$ were females [Table-2]. Headache is the commonest symptom in our study followed by vomiting, seizures and altered sensorium [Table-3]. Papilledema is seen in 16 cases. Focal neurological deficit is noted in 13 cases. Pregnancy and puerperium are the most common predisposing factor for cerebral venous thrombosis in our study [Table-4]. CVT due to Oral contraceptive pills, Dehydration, alcohol was seen in significant number of cases in our study. Infection such as Chronic Suppurative Otitis Media and sinusitis were seen in 3 cases. Superior Sagittal sinus is the commonest one involved in our study followed by transverse and sigmoid sinus [Table-5]. Deep Cerebral veins are involved in 5 cases and isolated cortical vein thrombosis seen in 3 cases. Associated parenchymal abnormalities such as Infarcts are seen in 24 cases in which areas of hemorrhage noted in 16 cases and diffusion restriction noted in 4 cases only [Table-6]. Anticoagulants such as Low molecular weight heparin was administered to all the patients immediately after diagnosis with symptomatic treatment such as Hydration, antiepileptic and antiedema measures. On 
follow up [Table-7], near complete resolution of thrombosis and clinical symptoms in most of the cases(13 cases). One patient with internal cerebral vein thrombosis and thalamic and Brain stem infarcts died.

\section{DISCUSSION}

Cerebral venous thrombosis is a relatively a rare type of stroke and potentially life threatening which requires early prompt diagnosis and immediate anticoagulation therapy. ${ }^{5}$

Venous infarcts are either due to thrombosis of the venous system or external compression. Subsequently, continuous rise in venous pressure causes cortical edema which on imaging seen as vasogenic edema. Further rise in venous pressure obstructs the arterial inflow leading to cytotoxic edema. Cerebral edema will exacerbate the venous obstruction leading to a vicious cycle of edema and further cerebral venous obstruction. ${ }^{6}$

Cerebral venous thrombosis commonly affects younger age group contrary to arterial stroke. In our study, majority of the cases were seen from 21-50 years of age with a peak incidence at $4^{\text {th }}$ decade. In a study by Khaladkar et al in 2014 described similar type of incidence with a maximum number cases (57.5\%) in from 21-40 years of age. ${ }^{4}$ Cerebral venous thrombosis can affect both the sexes, but there was mild female preponderance seen in our study showing $58 \%$ of the cases with cerebral venous thrombosis were females. In a study by Walecki J et al in 2015 showed similar type of results. ${ }^{3}$

Pregnancy and Puerperium are common transient prothrombotic states which may lead to Cerebral venous thrombosis. ${ }^{7}$ It is the commonest predisposing factor in our study contributing $20 \%$ of the cases. Thammishetti et al in 2016 found similar results in their study. ${ }^{8}$ Oral contraceptive pills and Alcohol also contributed major number of cases in our study. No definite predisposing factors were identified in 9 cases. In a study by Khaladkar et al in 2014 described that most of the cases of CVT were idiopathic. ${ }^{4}$

A study in 2013, Alvis-Miranda HR stated Cerebral venous thrombosis may have a wide range of clinical presentations, however headache was the most common presentation. ${ }^{9}$ In our study also, headache is the most common presentation comprising $82 \%$ of the cases. Seizures are one of the common presentation in cerebral venous thrombosis. According to a study by Mahale et al in 2016, $46 \%$ of the cases had presented with acute seizures. ${ }^{10}$ In our study seizures was noted in 21 cases (42\%) which is almost comparable with study by Mahale et al. ${ }^{10}$ Focal neurological deficits are not uncommon in cerebral venous thrombosis. Motor deficits are the commonest form of neurological deficits in patients with cerebral venous thrombosis. ${ }^{11}$ In our study, 13 patients had presented with neurological deficits. Cerebral Venous thrombosis may present with ophthalmic symptoms due to papilledema, loss of vision and constriction of visual field. ${ }^{11}$ In our study papilledema was seen in 16 cases(32\%) and visual disturbances noted in 5 cases.

Superior sagittal sinus (56\%) was the commonest one involved in our study, followed by transverse (48\%) and sigmoid (36\%) sinuses. Deep cerebral veins were involved in 5 cases $(10 \%)$. Similar type of involvement was seen in a study by Leach JL in $2006 .^{12}$

Unlike arterial occlusion, approximately $50 \%$ of the cases with cerebral venous thrombosis results in infarct in which $50 \%$ of the infarcts are reversible with anticoagulation therapy. ${ }^{6}$ In our study, parenchymal changes such as infarcts with/without hemorrhage were noted in 24 cases, amongst which only 4 cases showed diffusion restriction, 8 cases showed infarct without hemorrhage and 16 cases showed infarcts with hemorrhage. 28 cases showed no parenchymal changes.

In our study out of 50 patients, 20 patients came for regular follow up visits among which around $65 \%$ of the cases showed complete recovery without any neurological deficit. Rottger C et al in 2005 stated in his study that parenchymal changes are usually due to edema and even larger parenchymal lesion can resolve independent of recanalization of affected vein but mainly depends on the development of collateral veins. ${ }^{13}$ Patients with Infarcts showing diffusion restriction had permanent neurological deficits. In our study, 4 cases showed diffusion restriction in areas of infarct and all the four cases had residual neurological deficits.

\section{Limitation}

Sample size of our study is very less to assess the common predisposing factors. Proper Follow up and post treatment imaging is not available with all the cases in our study to check the clinical outcome.

\section{CONCLUSION}

Cerebral venous thrombosis is not an uncommon cause of stroke usually seen in adults. Relatively more common in females especially during pregnancy and puerperium and in those having oral contraceptive pills. Due to recent advancements in imaging, the diagnosis has been made easy. Also, prompt diagnosis allows the early anticoagulant administration which leads to complete clinical recovery without significant residual neurological deficits in patients with Cerebral venous thrombosis. MRI and MR venogram are more efficient in diagnosing the cerebral venous thrombosis and to assess the prognosis based on parenchymal changes.

\section{REFERENCES}

1. Goyal G, Charan A, Singh R. Clinical Presentation, Neuroimaging Findings, and Predictors of Brain Parenchymal Lesions in Cerebral Vein and Dural Sinus Thrombosis: A Retrospective Study. Ann Indian Acad Neurol. 2018;21(3):203-208.

2. Stam J, author. Thrombosis of the cerebral veins and sinuses. N Engl J Med. 2005;352(5):1791-8.

3. Walecki J, Mruk B, Nawrocka-Laskus E, Piliszek A, Przelaskowski A, Sklinda K. Neuroimaging of Cerebral Venous Thrombosis (CVT) - Old Dilemma and the New Diagnostic Methods. Pol J Radiol. 2015;80(1):368-73.

4. Khaladkar SM, Thakkar DK, Thakkar DK, Shrotri H, Kulkarni VM. Cerebral venous sinus thrombosis on MRI: A case series analysis. Med J D Y Patil Univ 2014;7(1):296-303.

5. Bonneville F. Imaging of cerebral venous thrombosis. Diagn Interv Imaging. 2014;95(4):1145-50.

6. Lu A, Shen PY, Dahlin BC, Nidecker AE, Nundkumar 
A, Lee PS. Cerebral venous thrombosis and infarct: Review of imaging manifestations. Appl Radiol. 2016;45(3):9-17.

7. Saposnik G, Barinagarrementeria F, Brown RD Jr, et al. Diagnosis and management of cerebral venous thrombosis: a statement for healthcare professionals from the American Heart Association/American Stroke Association. Stroke. 2011;42(4):1158-1192

8. Thammishetti V, Dharanipragada S, Basu D, Ananthakrishnan R, Surendiran D. A Prospective Study of the Clinical Profile, Outcome and Evaluation of D-dimer in Cerebral Venous Thrombosis. J Clin Diagn Res. 2016;10(6):OC07-10.

9. Alvis-Miranda HR, Milena Castellar-Leones S, Alcala-Cerra G, Rafael Moscote-Salazar L. Cerebral sinus venous thrombosis. J Neurosci Rural Pract. 2013;4(4):427-38.

10. Mahale R., Mehta A., John A. A., Buddaraju K., Shankar A. K., Javali M., et al. Acute seizures in cerebral venous sinus thrombosis: what predicts it? Epilepsy Res. 123, 1-5. 10.1016/j.eplepsyres. 2016.01.011.

11. Luo Y, Tian X, Wang X. Diagnosis and Treatment of Cerebral Venous Thrombosis: A Review. Front Aging Neurosci. 2018;10:2.

12. Leach JL, Fortuna RB, Jones BV, Gaskill-Shipley MF. Imaging of cerebral venous thrombosis: Current techniques, spectrum of findings, and diagnostic pitfalls. Radiographics. 2006;26(1):S19-41.

13. Röttger C, Trittmacher S, Gerriets $T$, et al. Reversible MR imaging abnormalities following cerebral venous thrombosis. AJNR Am J Neuroradiol 2005;26(3):60713.

Source of Support: Nil; Conflict of Interest: None

Submitted: 16-01-2019; Accepted: 19-02-2019; Published online: 13-03-2019 